\title{
Relationship between thermodynamic perturbation and scaled particle theories for fused dimers fluids
}

\author{
O. Bernard \\ Laboratoire PHENIX, CNRS, Sorbonne Université (Campus P.M. Curie), 4 Place Jussieu, \\ Case 51, F-75005 Paris, France
}

Received July 6, 2021, in final form August 2, 2021

\begin{abstract}
Various approaches are reviewed that use scaled particle theories to describe dumbbell fluids made of tangent or overlapped hard spheres. Expressions encountered in the literature are written in a form similar to that presented in the thermodynamic perturbation theory introduced by Wertheim for chains and developed in statistical associating fluid theory (SAFT). Analogies and differences observed in these two types of theoretical descriptions allow one to propose alternative theoretical expressions to describe dumbbell fluids with overlapping spheres.
\end{abstract}

Key words: hard dumbbells fluid, scaled particle theory, thermodynamic perturbation theory

\section{Introduction}

The description of thermodynamic properties of media containing non-spherical objects is still important in many fields of fundamental or applied science. It still presents difficulties, in particular in the description of phase transitions and the various types of phases generated according to the geometry of the objects. Convex objects were described previously using extensions of scaled particle theory (SPT), which was initially introduced for spheres [1]. These extensions, sometimes found using virial developments, have given rise to various conformal theories [2-5]. They have led in particular to free volume theories [6] used to describe phase transitions, as well as to some approaches of inhomogeneous fluids [7].

Furthermore, the chains of spheres linked together could also be described within the thermodynamic perturbation theory (TPT) proposed by Wertheim [8, 9], which led to the statistical associated fluid theory (SAFT) [10] and its extensions currently used to describe polymer fluids. This approach separates the thermodynamic quantities considered (free energy or pressure) into a reference contribution, related to the subunits in absence of connection, and into a contribution induced by the connectivity between these subunits necessary to form the chains. In addition, the spheres constituting the chains are most often constrained to be tangent because the description of spheres with overlap proved to be more difficult. However, in the extensions of the TPT used to describe polymers, it is often useful to be able to describe various subunits within chains as groups of spheres with overlap. Better consideration of such subunits with the scaled particle theory could be useful to improve the application of TPT to polymers.

On the other hand, chains of spheres cannot in principle be described with scaled particle theory or its variants because these objects are non-convex. However, the theoretical SPT models of spherocylinders have been adapted to describe linear chains. In particular, this has provided a good description of dumbbell fluids formed of two overlapping spheres. This is probably due to the similarity in shape between these dumbbells and slightly elongated spherocylinders. Another conformal theory, called scaled field particle (SFP) theory and having similarities with SPT, has also been used to describe dimer fluids. Then, in this work, these two versions of conformal theories were reviewed and applied to describe thermodynamics of dimer fluids. This paper is dedicated to Yu. V. Kalyuzhnyi, who has made significant contributions in many different research areas related to statistical mechanics, especially in integral equation theories. 
The reference contribution in TPT is that of dissociated hard spheres. Then, in the next section, the description of hard spheres and its use in TPT theory is presented. A theoretical expression of the dimer pressure obtained using integral equations is also given to later highlight links with the other approaches presented. In the following section, various conformal or free volume theories inspired by SPT are presented. Conformal theories describe the thermodynamic quantities of fluids by using geometric parameters characteristic of the bodies considered, such as their volumes, surface areas and mean radii. The two types of conformal theories studied do not use exactly the same set of parameters. In addition, for each type of conformal theory, from the original result a corrective term has been added to obtain an improved version. The corrective term depends also on the set of geometric parameters. Since the two types of conformal theories do not use exactly the same geometric parameters, the corrective term is different. So, for each theory we have presented both the original version and the improved version to be able to better study the influence of the corrective term.

At small separation between the spheres constituting the dimer, when the larger sphere tends to completely overlap the smaller one, it is known that the two types of conformal theories tend towards the same result. The difference between the results given by the two approaches increases with the elongation between the components of the dimer. Then, in the next section, in order to highlight the differences between the two conformal approaches, these theories were first applied to dimers made of tangent spheres. For this particular configuration, all of the above-mentioned geometric parameters can be expressed from the diameters of the hard spheres. Then, the thermodynamic quantities coming from the various conformal theories, were rewritten as a function of the diameters of the hard spheres instead of the aforementioned geometric parameters. In particular, when the hard spheres have all the same diameter, the pressure of the dimer fluid is described simply as a function of the volume fraction. Moreover, these expressions were split into two part according to the same dividing found in the TPT, namely a reference term of dissociated hard spheres and a connectivity term due to the links between spheres. This made it possible to better analyze the reference term and the connectivity term in each of the theories. This made it possible to explain the differences observed in the description of the thermodynamics deduced from simulations.

After this analysis of previous works, in the following section alternative relationships have been established to describe first dumbbells made of tangent spheres, and then those with overlap. A comparison was given with the results of simulations. In a last part we conclude this work and present the prospects that it suggests.

\section{From integral equations to perturbation theory for hard spheres and tangent hard sphere dimers}

\subsection{Integral equation for dissociated hard spheres fluid}

A proper account of the excluded volume of various species is essential to describe thermodynamic properties such as the pressure $P$ of liquids and concentrated solutions [11-14]. Since the sphere is the simplest object taking into account the repulsion at short distances, all the constituents were first considered as hard spheres. In addition, molecules of complex shape can be represented using collections of spheres linked together. Therefore, it seems essential to first properly describe the properties of these subunits constituting these assemblies. Numerical simulations make it possible to precisely determine the structural and thermodynamic properties of model systems. However, their implementation is cumbersome and does not lead to explicit expressions of the studied quantities. As an alternative, studies of the structural and thermodynamic properties of hard spheres were undertaken using the OrnsteinZernike (OZ) integral equations [15]. Among various closure relations useful in solving these integral equations, the Percus Yevick (PY) approximation [16] has proven particularly suitable to describe hard spheres. In addition, it provides analytical expressions of some structural and thermodynamic quantities. Notably, the PY approximation allows one to access the radial distribution functions $g_{i j}(r)$ between all pairs of components $i$ and $j$ present in the liquid [17-19]. Various thermodynamic quantities can be calculated from the radial distribution functions $g_{i j}(r)$ [20]. In particular, from the relation defining the compressibility as a function of these $g_{i j}(r)$, an analytic expression of the pressure has been deduced by 
integration [21-23]

$$
\beta P_{\mathrm{PY}}^{c}=\frac{6}{\pi}\left[\frac{\zeta_{0}}{1-\zeta_{3}}+\frac{3 \zeta_{1} \zeta_{2}}{\left(1-\zeta_{3}\right)^{2}}+\frac{3 \zeta_{2}^{3}}{\left(1-\zeta_{3}\right)^{3}}\right],
$$

where $\beta$ is equal to $1 / k_{\mathrm{B}} T$ with $k_{\mathrm{B}}$ the Boltzmann constant and $T$ the temperature. The terms $\zeta_{n}$ are given by

$$
\zeta_{n}=\frac{\pi}{6} \sum_{k} \rho_{k} \sigma_{k}^{n}
$$

where $\rho_{k}$ and $\sigma_{k}$ are the number density and the diameter of component $k$, respectively. Subscript "PY", under the symbol $P$, means that the pressure was obtained in the PY approximation, and the superscript " $c$ " has been added to remind that this expression comes from the compressibility relation. The virial relation also provides an analytical expression of the pressure $P_{\mathrm{PY}}^{v}$, which is however less precise in comparison with the results deduced from numerical simulations. An alternative expression of the pressure somewhat more accurate than that given by equation $(2.1)$, has been deduced by a combinaison of the pressure deduced from the virial and the compressibility relations, first in the case of a single component [24] and then for mixtures of several constituents [25, 26] :

$$
\beta P_{\mathrm{BM}}=\frac{6}{\pi}\left[\frac{\zeta_{0}}{1-\zeta_{3}}+\frac{3 \zeta_{1} \zeta_{2}}{\left(1-\zeta_{3}\right)^{2}}+\frac{3 \zeta_{2}^{3}-\zeta_{2}^{3} \zeta_{3}}{\left(1-\zeta_{3}\right)^{3}}\right] .
$$

The subscript "BM" means Boublík-Mansoori, to recall the initials of the two first authors of the articles in which were established the corresponding expression for mixtures of several constituents [25, 26]. The "BMCSL" subscript (or superscript) is often used to denote this equation, to recall the initials of all the authors who established it for mixture of components [25, 26]. Here, we limited the number of letters in subscript to alleviate the notation. Expressions giving an even better description of the pressure obtained by simulation were proposed later. However, in this article we limit ourselves to this level of refinement, compared to the expression (2.1) determined using the PY approximation. Indeed, the passage of equation (2.1) to (2.3) is simple enough to be used later in the context of expressions describing non-spherical objects. Moreover, equation (2.3) is commonly used both in perturbation theories and in applications to describe experimental systems. Thereafter, from these expressions of the pressure, other quantities, such as the free energy $A$ or the chemical potentials $\mu_{j}$ of various $j$ constituents, can be deduced by integration or differentiation. They will also be useful when applying perturbation theories which take the fluid of dissociated hard spheres as a reference.

\subsection{Integral equations for dimers made of tangent hard spheres}

Model molecules, made of assemblies of hard spheres were considered. Dimers made of hard tangent spheres are the simplest of these aggregates. In order to describe dimers with integral equations, attractive sites were added on their surfaces. These sites induce the formation of bonds between the spheres. If there is only one site per sphere, only dimers can be formed. Since the sites occupying a very small fraction of the surface of the spheres, the attractive interactions are highly directional. Dimers are formed only when two contacting spheres have their sites oriented towards each other. In the other configurations, particles interact only with the purely radial potential (hard spheres potential). The consideration of these highly directional potentials permits using various integral equations to describe the structure and interactions of these hard sphere assemblies [8, 9, 27,-33]. In particular, Wertheim's OZ equations (WOZ) [8, 9, 29,-32] were applied to the description of dimers formed by tangent spheres [31, 34-36]. As an extension of the PY relation, a polymer Percus Yevick (PPY) closure relation was used. Moreover, the density $\rho_{i}$ of various $i$ species, is decomposed into the density of free $i$ species $\rho_{i}^{0}$, and dimers $\rho_{\mathrm{D}}$. Considering, for simplicity only, two species 1 and 2 which can link together to form dimers, we have the mass conservation relations, for $i=1$ or 2 ,

$$
\rho_{i}=\rho_{i}^{0}+\rho_{\mathrm{D}}
$$

with

$$
\rho_{\mathrm{D}}=\rho_{1}^{0} \rho_{2}^{0} K^{0} g_{12}^{00}\left(\sigma_{12}\right)
$$


where $K^{0}$ is an association constant (which can be expressed as an integral dependent on the attractive potential [31]), and $g_{12}^{00}\left(\sigma_{12}\right)$ is the pair distribution between unassociated species 1 and 2 at a contact distance $\sigma_{12}=\left(\sigma_{1}+\sigma_{2}\right) / 2$. The solution of the WOZ equations allows one to accurately describe the interactions between these constituents. As in the case of the dissociated hard spheres, analytic expressions of the pressure, deduced from the virial and compressibility relations, were obtained. Here again, the expression deduced from the compressibility is the most efficient [31, 36].

$$
\begin{aligned}
\beta P_{\mathrm{PPY}}^{c} & =\beta P_{\mathrm{PY}}^{c}-\rho_{1}^{0} \rho_{2}^{0} K^{0}\left[g_{12}^{00}\left(\sigma_{12}\right)\right]^{2} \\
& =\beta P_{\mathrm{PY}}^{c}-\rho_{\mathrm{D}} g_{12}^{00}\left(\sigma_{12}\right) .
\end{aligned}
$$

The definition of $\rho_{\mathrm{D}}$, given by the equation (2.5), has been used to go from equation (2.6a) to 2.6b). Furthermore, in the PPY approximation, it was found that $g_{12}^{00}\left(\sigma_{12}\right)$ is equal to $g_{12}^{\mathrm{PY}}\left(\sigma_{12}\right)$ [31, 35], which is the corresponding pair distribution function at contact, obtained in the PY approximation for hard spheres without attractive sites. Moreover, $g_{12}^{\mathrm{PY}}\left(\sigma_{12}\right)$ is given by [19]

$$
g_{12}^{\mathrm{PY}}\left(\sigma_{12}\right)=\frac{1}{1-\zeta_{3}}+\frac{3 \zeta_{2}}{\left(1-\zeta_{3}\right)^{2}} \frac{\sigma_{1} \sigma_{2}}{\sigma_{1}+\sigma_{2}} .
$$

Equation 2.6b was also established within various integral equations devoted to the description of properties of chain fluids [37-40]. As a generalisation in equation 2.6b), for chains of density $\rho_{c}$ containing $m$ subunits, $\rho_{\mathrm{D}}$ is replaced by $(m-1) \rho_{c}$. In the next section, when comparing the various approaches, we come back to the explicit expression 2.6b derived from the compressibility relation within the PPY approximation.

\subsection{Thermodynamic perturbation theory for dimers made of tangent hard spheres}

As an alternative, a thermodynamic perturbation theory (TPT) was also developed to take into account the effect of the bonds formed between the particles on the thermodynamic properties of the fluid [8, 9, 29-32]. The generalization and application of this theoretical path in the SAFT led to one of the most accurate equations of state for tangent hard sphere chains [10, 41-43]. These approaches describe the thermodynamics in terms of the reference fluid distribution functions regardless of the amount of bonding. The residual Helmholtz free energy $A$ can be separated into two terms as follows:

$$
\frac{\beta A_{\mathrm{TPT}}}{V}=\frac{\beta A^{\mathrm{ref}}}{V}+\frac{\beta A^{\text {bond }}}{V},
$$

where $A^{\text {ref }}$ is the reference Helmholtz free energy contribution from the dissociated free monomers, and $A^{\text {bond }}$ is a contribution related to the formation of bonds. In the 1 st order perturbation theory, the term $A^{\text {bond }}$ can also be evaluated from the properties of the reference system without association.

$$
\frac{\beta A_{\mathrm{TPT}}}{V}=\frac{\beta A^{\mathrm{ref}}}{V}-(m-1) \rho_{c} \ln g^{\mathrm{ref}}\left(\sigma_{12}\right) .
$$

In the second term of equation (2.9), related to the connectivity within the chains, $m$ is the number of monomers, $\rho_{c}$ the number density of the chain and $g^{\text {ref }}\left(\sigma_{12}\right)$ the pair distribution function between two monomers at a distance of contact $\sigma_{12}$ in the reference fluid without association. For simplicity, it is assumed here that all the monomers are separated by the same contact distance in the chains. If the reference fluid consists of hard spheres, the reference free energy can be described using either the PY expression,

$$
\frac{\beta A_{\mathrm{PY}}^{c}}{V}=\frac{6}{\pi}\left[-\zeta_{0} \ln \left(1-\zeta_{3}\right)+\frac{3 \zeta_{1} \zeta_{2}}{1-\zeta_{3}}+\frac{3 \zeta_{2}^{3}}{2\left(1-\zeta_{3}\right)^{2}}\right],
$$


corresponding to the expression of the pressure given by equation (2.1) or the improved expression associated with the pressure given by 2.3 ,

$$
\frac{\beta A_{\mathrm{BM}}}{V}=\frac{6}{\pi}\left[\left(\frac{\zeta_{2}^{3}}{\zeta_{3}^{2}}-\zeta_{0}\right) \ln \left(1-\zeta_{3}\right)+\frac{3 \zeta_{1} \zeta_{2}}{1-\zeta_{3}}+\frac{\zeta_{2}^{3}}{\zeta_{3}\left(1-\zeta_{3}\right)^{2}}\right] .
$$

This latter expression is the one generally used because it leads to the results closest to those given by the simulations, in comparison with those given by equation $(2.10)$. However, we have also presented expression 2.10 , deduced from the PY theory, because it can also be recovered using other theories presented below in this article. This property will be used later to develop new improved expressions. In the second term of equation (2.9), the pair distribution function at contact can be described either using an expression deduced from the $\mathrm{OZ}$ equations, or using an expression corresponding to the improved expression of pressure 2.3. The pressure $P_{\mathrm{TPT}}=P^{\text {ref }}+P^{\text {bond }}$ can be deduced from equation 2.9 by differentiation

$$
\beta P_{\mathrm{TPT}}=\beta P^{\mathrm{ref}}-(m-1) \rho_{c}\left[1+\sum_{k} \rho_{k} \frac{\partial \ln g^{\mathrm{ref}}\left(\sigma_{12}\right)}{\partial \rho_{k}}\right] .
$$

In the same way, when the reference fluid consists of hard spheres, the reference pressure $P^{\text {ref }}$ can be described either using the PY expression (2.1), or the improved expression (2.3). It is recalled that in the case where only dimers are formed, $m=2$, the concentration of chains $\rho_{c}$ denotes the concentration of dimers $\rho_{\mathrm{D}}$. Then, in equations 2.9 ) and 2.12), $(m-1) \rho_{c}$ can be simply replaced by $\rho_{\mathrm{D}}$.

Attempts have been made to extend TPT to fluids of fused sphere chains [44-47]. In equations (2.9]) and 2.12), the distribution function $g^{\text {ref }}\left(\sigma_{12}\right)$ is replaced by a cavity correlation function $y(l)$ where $l \leqslant \sigma_{12}$, is the distance between the spheres linked together. Moreover, an effective number of tangent monomers was used by requiring that the second virial coefficient of the chain of tangent spheres should be equal to that of the chain of fused spheres. In the case of fused spheres, this second virial coefficient can be expressed in terms of parameters of non-sphericity which account for the molecular shape of the chain. This approach is based on the contribution of various conformal theories describing the properties of objects of various shapes.

\section{Conformal or free volume theories}

One of the goals of conformal or free volume theories is to attempt to describe individual chemical potentials and an equation of state of convex body in terms of a relevant set of parameters such as the volume, surface area and the mean radius of curvature which characterize the shape of all the components. This approach was first performed in the scaled particle theory (SPT).

\subsection{Scaled particle theory}

The SPT was first developed to describe the hard sphere fluids [1, 48]. Analytical expressions of the radial distribution functions at contact and of thermodynamic quantities were obtained. It is noted, that the resulting expression of the pressure is identical to that given in equation $(2.1)$, derived from the compressibility relation and the PY approximation. The SPT was extended to the description of convex objects other than hard spheres, such as ellipsoids and spherocylinders. In particular, the studies of prolate spherocylinders led to an analytical description of the pressure [49-51]

$$
\beta P_{\mathrm{SPT}}=\frac{\rho}{1-v}+\frac{r s}{(1-v)^{2}}+\frac{q s^{2}}{3(1-v)^{3}},
$$

where:

$$
\rho=\sum_{i} \rho_{i}, \quad v=\sum_{i} \rho_{i} V_{i}, \quad s=\sum_{i} \rho_{i} S_{i}, \quad r=\sum_{i} \rho_{i} R_{i} \text { and } q=\sum_{i} \rho_{i} R_{i}^{2} .
$$

This result for $P$, is no longer only a function of the diameters of the spheres, but is also expressed as a function of the volume $V_{i}$, of the surface area $S_{i}$ and of the mean curvature $R_{i}$ of each $i$ object. This 
formula is a generalization of that obtained within SPT for mixtures of hard spheres. In this case, the geometric parameters are related to the diameters $\sigma_{i}: R_{i}=\sigma_{i} / 2, S_{i}=\pi \sigma_{i}^{2}$ and $V_{i}=(\pi / 6) \sigma_{i}^{3}$. Using these relations in equation (3.1), leds to the relation (2.1), previously deduced from the compressibility in the PY approximation.

An improved scale particle theory (ISPT) was developed as a generalization of equation (2.3), and a more accurate expression of the pressure was obtained [2]

$$
\beta P_{\mathrm{ISPT}}=\frac{\rho}{1-v}+\frac{r s}{(1-v)^{2}}+\frac{q s^{2}(3-v)}{9(1-v)^{3}} .
$$

Here also, when all the constituents are spherical, this formula becomes identical to that given by equation (2.3). In principle, SPT is only applicable to convex objects. Dumbbells resemble prolate spherocylinders but are not convex and the result of the SPT should not be used for these model molecules. Nevertheless, the previous equation (3.2) was applied to fused dimers [52] and the results agree well with the simulation data [53]. Otherwise, equation (3.1) leads to an exact second virial coefficient and to an approximate third coefficient. On the other hand, equation (3.2) provides a third virial coefficient closer to the exact value. It is noted that the different values found for the third coefficient seems to be related to the difference observed between the third terms to the right of the equality in each of these two equations. Moreover, there are other theoretical developments attempting to jointly describe the thermodynamics and the structure of fluids of convex objects.

\subsection{Scaled field particle theory}

Scaled field particle (SFP) theory provides an explicit expression of direct correlation functions in addition to the quantities also determined by SPT [4]. The expressions of various thermodynamic quantities are very close to those deduced within the SPT. In particular, the pressure is written with an expression very close to equation (3.1), derived under the SPT:

$$
\beta P_{\mathrm{SFP}}=\frac{\rho}{1-v}+\frac{r s}{(1-v)^{2}}+\frac{1}{12 \pi} \frac{s^{3}}{(1-v)^{3}} .
$$

This formula differs only in the last term, where $q s^{2}$ is replaced by $s^{3} /(4 \pi)$.

In the same way, an improved scale field relation (ISFP) was proposed as a generalization of that leading to equation (2.3). This relation can be easily deduced from equation (3.2), by replacing, in the third term to the right of the equality, $q s^{2}$ by $s^{3} /(4 \pi)$.

$$
\beta P_{\mathrm{ISPP}}=\frac{\rho}{1-v}+\frac{r s}{(1-v)^{2}}+\frac{s^{3}(3-v)}{36 \pi(1-v)^{3}} .
$$

From the SPT and the SFP, we have introduced four possible expressions to account for the pressure variations observed in a fluid of convex objects. In the following sections, a comparison between these various relationships is presented, when used to describe a fluid of tangent or fused hard dimers. New expressions are also established based on the mathematical form of the TPT equations [2.9] and (2.12).

\section{Application of conformal theories to dumbbells}

A comparison of the results obtained using various conformal theories introduced in the previous section may be done, when the fluid is made of dumbbells. Dumbbells made of two spheres, denoted by indices 1 and 2, of diameters $\sigma_{1}$ and $\sigma_{2}$, separated by a distance: $l \leqslant \sigma_{12} \equiv\left(\sigma_{1}+\sigma_{2}\right) / 2$, are considered. The characteristic quantities $V_{12}, S_{12}$ and $R_{12}$ of these objects are [4, 46, 47]

$$
V_{12}=\frac{\pi}{12}\left[\sigma_{1}^{3}+\sigma_{2}^{3}+\frac{3}{2}\left(\sigma_{1}^{2}+\sigma_{2}^{2}\right) l+\frac{3}{16} \frac{\left(\sigma_{1}^{2}-\sigma_{2}^{2}\right)^{2}}{l}-l^{3}\right],
$$




$$
\begin{gathered}
S_{12}=\frac{\pi}{2}\left[\sigma_{1}^{2}+\sigma_{2}^{2}+\left(\sigma_{1}+\sigma_{2}\right)\left(l+\frac{\left(\sigma_{1}-\sigma_{2}\right)^{2}}{4 l}\right)\right], \\
R_{12}=\frac{1}{4}\left[\sigma_{1}+\sigma_{2}+l+\frac{\left(\sigma_{1}-\sigma_{2}\right)^{2}}{4 l}\right] .
\end{gathered}
$$

Assuming that $\sigma_{1}$ is greater than $\sigma_{2}$, one can first determine the values taken by these quantities when $l \leqslant\left(\sigma_{1}-\sigma_{2}\right) / 2$. In this case, sphere 2 is completely included in sphere 1 and various geometric quantities defined above take the values corresponding to sphere 1 alone. Once these quantities are known, when only dumbbells are present (with $\rho_{1}=\rho_{2}$ ), the pressure can be determined. It is found that the expressions of $P_{\mathrm{SPT}}$ and $P_{\mathrm{SFP}}$ are identical to $P_{\mathrm{PY}}^{c}$, given by equation $\sqrt{2.1}$, with $\zeta_{n}=(\pi / 6) \rho_{1} \sigma_{1}^{n}$ and $n=0,1,2$ and 3 . In the same way, the expressions of $P_{\mathrm{ISPT}}$ and $P_{\mathrm{ISFP}}$ are identical to $P_{\mathrm{BM}}$, given by equation (2.3). Then, when $l \leqslant\left(\sigma_{1}-\sigma_{2}\right) / 2$, SPT and SFP expressions of the pressure merge to the PY compressibility relation for hard spheres, and ISPT and ISFP expressions merge to the improved BMCSL for hard spheres.

The limit where $l=\sigma_{12}$ is also very important. Again, in this case the various geometric quantities can be explained in terms of the diameters of the spheres only. The thermodynamics deduced from conformal theories leads to relations similar to those obtained with TPT. These relationships are presented in the following subsections.

\subsection{Pressure for dimers made of tangent hard spheres}

When the two spheres are in contact: $l=\sigma_{12}$, then $V_{12}=(\pi / 6)\left(\sigma_{1}^{3}+\sigma_{2}^{3}\right)$ and $S_{12}=\pi\left(\sigma_{1}^{2}+\sigma_{2}^{2}\right)$, are the sum of the volumes and surface areas of the two spheres, respectively. However, the quantity $R_{12}$ is different from the sum of the two separate radii, namely $R_{12}=\left(\sigma_{1}+\sigma_{2}\right) / 2-\sigma_{1} \sigma_{2} /\left[2\left(\sigma_{1}+\sigma_{2}\right)\right]$.

Now, in the case of a dimer formation equilibrium, let us denote by $\rho_{1}^{0}$ and $\rho_{2}^{0}$, the densities of fully dissociated particles 1 and 2 , which can form dumbbells $\mathrm{D}$, of number density $\rho_{\mathrm{D}}$, with the equilibrium

$$
(1)+(2) \rightleftharpoons(D) .
$$

With this chemical equilibrium, it is observed that the concentrations that naturally occur are $\rho_{1}^{0}, \rho_{2}^{0}$ and $\rho_{\mathrm{D}}$ and not directly $\rho_{1}$ and $\rho_{2}$, the total concentrations (free or bound) of particles 1 and 2 . Then, from the SPT equations, the pressure $P_{\mathrm{SPT}}$ can be evaluated by considering the fluid as a mixture of two kinds of hard spheres components, of densities $\rho_{1}^{0}$ and $\rho_{2}^{0}$, and dimers, of density $\rho_{\mathrm{D}}$. The geometric parameters, $R_{i}, S_{i}, V_{i}$, of these two kinds of an object can be determined thanks to the equations (4.1)(4.3). The introduction of equilibrium makes it possible to link the chemical potentials of free and linked species. In addition, the quantities of the three species involved in equilibrium are related by the mass conservation relations (2.4). It has been noted previously [54, 55] that the thermodynamic relationships for systems with association can be described equivalently, either with the densities of the various species in equilibrium or with the total densities of species 1 and 2, considering $\rho_{\mathrm{D}}$ the density of pairs as a function of total densities. In the case of dumbbells made of tangent spheres, the integral equations within the PPY approximation and the perturbation theory lead to expressions which are functions of the total densities and of the density of dimers. Then, from the conformal expressions $\sqrt{3.1}-\sqrt{3.3})$, equations are established which are also expressed as a function of the total and dimer densities.

Thus, in the SPT equation $\sqrt{3.1}$ with three constituents, one can first replace the densities $\rho_{1}^{0}$ and $\rho_{2}^{0}$ by $\rho_{1}-\rho_{\mathrm{D}}$ and $\rho_{2}-\rho_{\mathrm{D}}$, respectively, in the quantities $\rho, r, s, q$ and $v$. One then obtains, when $l=\sigma_{12}$

$$
\beta P_{\mathrm{SPT}}=\beta P_{\mathrm{PY}}^{c}-\rho_{\mathrm{D}}\left[g_{12}^{\mathrm{PY}}\left(\sigma_{12}\right)-\frac{3 \zeta_{2}^{2}}{\left(1-\zeta_{3}\right)^{3}} \frac{\sigma_{1}^{2} \sigma_{2}^{2}}{\left(\sigma_{1}+\sigma_{2}\right)^{2}}\right],
$$

where $g_{12}^{\mathrm{PY}}\left(\sigma_{12}\right)$ is given by equation 2.7$)$. In the equation 4.5 , the terms $\zeta_{n}(n=0, \ldots, 3)$, appearing in $\beta P_{\mathrm{PY}}^{c}$ and in the second part of the equation, are calculated by considering the total concentrations $\rho_{1}$ and $\rho_{2}$ of the two constituents :

$$
\zeta_{n}=\frac{\pi}{6}\left(\rho_{1} \sigma_{1}^{n}+\rho_{2} \sigma_{2}^{n}\right)
$$




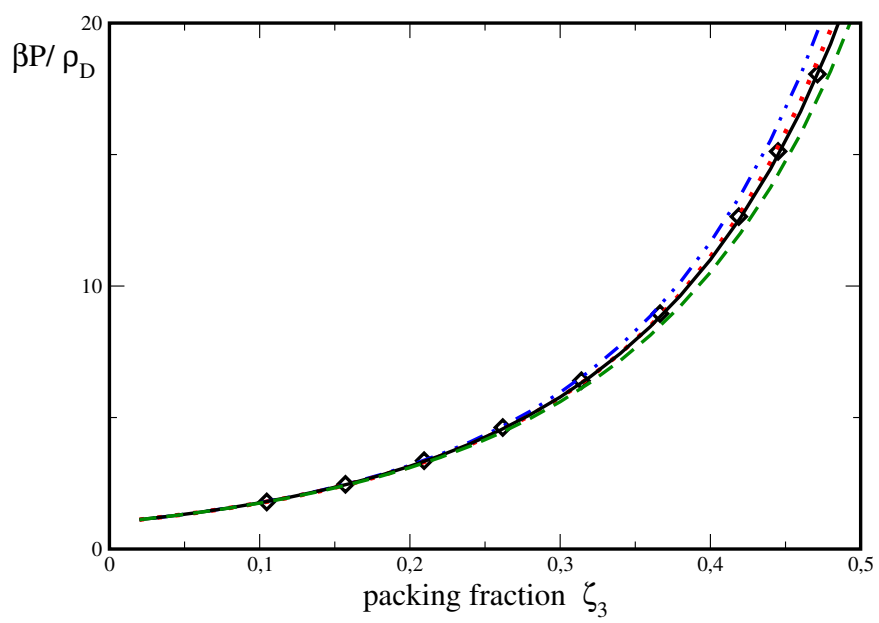

Figure 1. (Colour online) Equation of state for tangent hard sphere dumbbells. Lines from top to bottom represent, respectively, - - S SPT equation 4.5, $\cdots$ SFP equation (4.8), - ISPT equation (4.7), - - - ISFP equation 44.9]. Diamonds are simulation results (from [53]).

In the same way, for the improved SPT, equation (3.2) led to

$$
\beta P_{\mathrm{ISPT}}=\beta P_{\mathrm{BM}}-\rho_{\mathrm{D}}\left[g_{12}^{\mathrm{PY}}\left(\sigma_{12}\right)-\frac{\zeta_{2}^{2}\left(3-\zeta_{3}\right)}{\left(1-\zeta_{3}\right)^{3}} \frac{\sigma_{1}^{2} \sigma_{2}^{2}}{\left(\sigma_{1}+\sigma_{2}\right)^{2}}\right] .
$$

The same procedure can be applied to expressions deduced from the SFP theories. When $l=\sigma_{12}$, equation (3.3) led to

$$
\beta P_{\mathrm{SFP}}=\beta P_{\mathrm{PY}}^{c}-\rho_{\mathrm{D}} g_{12}^{\mathrm{PY}}\left(\sigma_{12}\right) .
$$

Similarly, from the equation for the improved SFP theory, one gets

$$
\beta P_{\mathrm{ISFP}}=\beta P_{\mathrm{BM}}-\rho_{\mathrm{D}} g_{12}^{\mathrm{PY}}\left(\sigma_{12}\right) .
$$

Note that the four equations 4.5, (4.7), 4.8, 4.9) have a mathematical form similar to that of the TPT equation given by 2.12). Indeed, they are all composed of a first term, related to the dissociated hard spheres, and a second term due to the connectivity between the spheres. By analogy with the equation 2.12, the first pressure term can be regarded as $\beta P^{\text {ref }}$, the reference pressure, and the second term as $\beta P^{\text {bond }}$, the contribution due to the formation of bonds. Apart from the proportionality to $\rho_{\mathrm{D}}$, the connectivity term only depends on the total densities of the hard spheres. In addition, the reference pressure is given by the PY expression in the original SPT and SFP theories. As expected, in the equations deduced from the improved theories, the reference pressure is given by the BM expression. We notice that in SPT theories the term of connectivity is different between the original version and the improved one. On the contrary, the connectivity term is the same in the original SFP theory and in the improved one. Moreover, the expression (4.8), deduced from the original SFP theory, is identical to that given by (2.6b), which was established using integral equations. Equation 4.9 was also proposed as an alternative to equation (4.8) within the framework of chain theory [37].

The results of the calculations carried out with these various formulae, for tangent homonuclear dimers (i.e., with a distance between its constituents: $l=\sigma_{12}$ ), are shown in figure 1 . As it has already been said before, the curve deduced from the improved theory ISPT with equation (3.2) is closest to the simulation results. Then, by comparing the initial expressions 3.1) and 3.3 obtained with SPT and SFP respectively, it is seen that they give values that are too high but that the latter is closer to the simulated values. Finally, the improved version ISFP gives values that are too low. 


\subsection{Connection with TPT deduced from free energy}

In the previous subsection, it was shown how, from the conformal equations $(3.1)-(3.3)$, applied to dumbbells formed by tangent spheres, the pressure can be split into a term of hard spheres repulsion and a term of connectivity between these spheres. This separation is similar to that encountered in the expression 2.12 of pressure in the TPT theory. Now, to further the analogy between the two kinds of description, we study the equations describing the free energy in conformal theories. The excess free energy can be deduced from the pressure by integrating the densities in each of the approximations presented above. Thereby, starting from equation 3.1 for $P_{\mathrm{SPT}}$, we find within the SPT

$$
\beta \frac{A_{\mathrm{SPT}}}{V}=-\rho \ln (1-v)+\frac{r s}{1-v}+\frac{q s^{2}}{6(1-v)^{2}} .
$$

In the same way, the free energy associated with the $P_{\text {ISPT }}$ pressure is given by:

$$
\beta \frac{A_{\mathrm{ISPT}}}{V}=\left(\frac{q s^{2}}{9 v^{2}}-\rho\right) \ln (1-v)+\frac{r s}{1-v}+\frac{q s^{2}}{9 v(1-v)^{2}} .
$$

As previously, the free energies $A_{\mathrm{SFP}}$, related to the pressure $P_{\mathrm{SFP}}$, and $A_{\mathrm{ISFP}}$, related to the pressure $P_{\mathrm{ISFP}}$, can be deduced from the two preceding equations by replacing everywhere $q s^{2}$ by $s^{3} /(4 \pi)$.

Now, the explicit relations of free energy, devoted to dimers formed of tangent spheres are presented. The mixture of dimers of density $\rho_{\mathrm{D}}$ and of the two dissociated monomers of density $\rho_{1}^{0}$ and $\rho_{2}^{0}$ is consider again. As previously, using mass conservation, $\rho_{1}^{0}$ and $\rho_{2}^{0}$ are expressed as a function of the total concentrations $\rho_{1}$ and $\rho_{2}$ and of the dimer concentration, namely, $\rho_{1}^{0}=\rho_{1}-\rho_{\mathrm{D}}$ and $\rho_{2}^{0}=\rho_{2}-\rho_{\mathrm{D}}$.

Equations (4.10) and 4.11) of the SPT theory are considered first. From the original relation (4.10), when $l=\sigma_{12}$, the application of the mass conservation leads to

$$
\beta \frac{A_{\mathrm{SPT}}\left(l=\sigma_{12}\right)}{V}=\beta \frac{A_{\mathrm{PY}}^{c}}{V}-\rho_{\mathrm{D}} \ln \mathcal{G}_{\mathrm{SPT}}\left(\sigma_{12}\right),
$$

where $A_{\mathrm{PY}}^{c}$ is given by the equation 2.10 and

$$
\ln \mathcal{G}_{\mathrm{SPT}}\left(\sigma_{12}\right)=-\ln \left(1-\zeta_{3}\right)+\frac{3 \zeta_{2}}{1-\zeta_{3}} \frac{\sigma_{1} \sigma_{2}}{\sigma_{1}+\sigma_{2}}-\frac{3 \zeta_{2}^{2}}{2\left(1-\zeta_{3}\right)^{2}} \frac{\sigma_{1}^{2} \sigma_{2}^{2}}{\left(\sigma_{1}+\sigma_{2}\right)^{2}} .
$$

As previously, in the expression of $A_{\mathrm{PY}}^{c}$ and $\ln \mathcal{G}_{\mathrm{SPT}}\left(\sigma_{12}\right)$, appearing in equations $(4.12)$ and $(4.13)$, the terms $\zeta_{n}$ (with $n=0, \ldots, 3$ ) are calculated with equation (4.6) by considering only the total concentrations $\rho_{1}$ and $\rho_{2}$ of the two constituents. A similarity can be seen between the form of equation (4.12) and that of equation 2.9), describing $A_{\mathrm{TPT}}$, the TPT excess free energy. The first term, to the right of the equality in the equation 4.12 , , corresponds to the reference free energy $A^{\text {ref }}$ in the equation $(2.9)$, when the PY approximation is chosen as the reference. Then, the second term in equation 4.12, corresponds to the contribution due to the connectivity between the hard spheres. We recall that in the TPT equation, the second term, proportional to the density of dimers $\rho_{\mathrm{D}}$, otherwise, depends only on the densities of dissociated spheres. We observe that the second term of the equation presents the same characteristics. If the analogy with the TPT equation is relevant, then the second term depends on $\ln g^{\text {ref }}\left(\sigma_{12}\right)$. We considered that the second term of the equation (4.12) had this meaning. That is why we gave it the name in relation $\ln \mathcal{G}\left(\sigma_{12}\right)$. Furthermore, within the framework of the SPT approximation applied to hard spheres, an explicit expression of the pair distribution function at contact, that we named $g_{12}^{\text {sPT }}\left(\sigma_{12}\right)$, was deduced previously [21].

$$
g_{12}^{\mathrm{SPT}}\left(\sigma_{12}\right)=\frac{1}{1-\zeta_{3}}+\frac{3 \zeta_{2}}{\left(1-\zeta_{3}\right)^{2}} \frac{\sigma_{1} \sigma_{2}}{\sigma_{1}+\sigma_{2}}+\frac{3 \zeta_{2}^{2}}{\left(1-\zeta_{3}\right)^{3}} \frac{\sigma_{1}^{2} \sigma_{2}^{2}}{\left(\sigma_{1}+\sigma_{2}\right)^{2}} .
$$

Then, the logarithm of $g_{12}^{\mathrm{SPT}}\left(\sigma_{12}\right)$ can be written as

$$
\ln \left[g_{12}^{\mathrm{SPT}}\left(\sigma_{12}\right)\right]=-\ln \left(1-\zeta_{3}\right)+\ln \left[1+\frac{3 \zeta_{2}}{\left(1-\zeta_{3}\right)} \frac{\sigma_{1} \sigma_{2}}{\sigma_{1}+\sigma_{2}}+\frac{3 \zeta_{2}^{2}}{\left(1-\zeta_{3}\right)^{2}} \frac{\sigma_{1}^{2} \sigma_{2}^{2}}{\left(\sigma_{1}+\sigma_{2}\right)^{2}}\right] .
$$


It was found that equation 4.13 for $\ln \mathcal{G}_{\mathrm{SPT}}\left(\sigma_{12}\right)$ can be recovered by taking a limited development to the second order in $\zeta_{2}$ of this expression.

Next, by applying the same process to the free energy $A_{\mathrm{ISPT}}$ of the improved SPT, when $l=\sigma_{12}$, it was found

$$
\beta \frac{A_{\mathrm{ISPT}}\left(l=\sigma_{12}\right)}{V}=\beta \frac{A_{\mathrm{BM}}}{V}-\rho_{\mathrm{D}} \ln \mathcal{G}_{\mathrm{ISPT}}\left(\sigma_{12}\right)
$$

with

$$
\ln \mathcal{G}_{\mathrm{ISPT}}\left(\sigma_{12}\right)=-\ln \left(1-\zeta_{3}\right)+\frac{3 \zeta_{2}}{1-\zeta_{3}} \frac{\sigma_{1} \sigma_{2}}{\sigma_{1}+\sigma_{2}}-\left(\ln \left(1-\zeta_{3}\right)+\frac{\zeta_{3}}{\left(1-\zeta_{3}\right)^{2}}\right) \frac{\zeta_{2}^{2}}{\zeta_{3}^{2}} \frac{\sigma_{1}^{2} \sigma_{2}^{2}}{\left(\sigma_{1}+\sigma_{2}\right)^{2}} .
$$

The first term, to the right of the equality in the equation (4.16), corresponds to the reference free energy $A^{\text {ref }}$ in the equation 2.9 , when the BM approximation is chosen as the reference.

As an alternative, the same process can be applied to the free energy expressions of the SFP theory. From the original expression 4.10 , by replacing $q s^{2}$ by $s^{3} /(4 \pi)$, when $l=\sigma_{12}$, one gets

$$
\beta \frac{A_{\mathrm{SFP}}\left(l=\sigma_{12}\right)}{V}=\beta \frac{A_{\mathrm{PY}}^{c}}{V}-\rho_{\mathrm{D}} \ln \mathcal{G}_{\mathrm{SFP}}\left(\sigma_{12}\right),
$$

where $\ln \mathcal{G}_{\mathrm{SFP}}\left(\sigma_{12}\right)$ is given by

$$
\ln \mathcal{G}_{\mathrm{SFP}}\left(\sigma_{12}\right)=-\ln \left(1-\zeta_{3}\right)+\frac{3 \zeta_{2}}{1-\zeta_{3}} \frac{\sigma_{1} \sigma_{2}}{\sigma_{1}+\sigma_{2}}
$$

Now, the same type of comparison can also be made between the original SFP and the improved SFP equation. By following again the same process, starting from equation 4.16] [but replacing $q s^{2}$ by $\left.s^{3} /(4 \pi)\right]$, when $l=\sigma_{12}$, it was found

$$
\beta \frac{A_{\mathrm{ISFP}}\left(l=\sigma_{12}\right)}{V}=\beta \frac{A_{\mathrm{BM}}}{V}-\rho_{\mathrm{D}} \ln \mathcal{G}_{\mathrm{SFP}}\left(\sigma_{12}\right) .
$$

As in the case of the SPT theory, during the transition from the original SFP expression to the improved one, it is seen that, in the first term, the free energy $A_{\mathrm{PY}}^{c}$ coming from the PY approximation is replaced by the improved $A_{\mathrm{BM}}$ equation. On the other hand, contrary to what had been found in the SPT theory, the second term remains unchanged during the transition from the original SFP relation to the improved one. In view of the numerical results presented in figure 1, the expressions (4.16) of the free energy lead by differentiation to a value of the pressure $P_{\text {ISPT }}$ that is much better than the one deduced from equation 4.20 in the ISFP theory. Since the first term $A_{\mathrm{BM}}$ due to the dissociated hard spheres is the same, in equations (4.16) and 4.20), there is a reason to believe that the difference observed in the resulting pressures comes from the connectivity terms $\ln \mathcal{G}\left(\sigma_{12}\right)$. A comparison between these various expressions of $\ln \mathcal{G}\left(\sigma_{12}\right)$ as a function of the volume fraction is presented in table 1 . It is observed that equations 4.13) and (4.17) give results of the same order of magnitude for the term $\ln \mathcal{G}\left(\sigma_{12}\right)$, unlike the SFP equation (4.19). In addition, equations (4.13) and (4.17), for $\ln \mathcal{G}_{\mathrm{SPT}}\left(\sigma_{12}\right)$ and $\ln \mathcal{G}_{\mathrm{ISPT}}\left(\sigma_{12}\right)$, respectively, give the values very close to those for $\ln g_{12}^{\mathrm{SPT}}\left(\sigma_{12}\right)$, deduced from equation 4.14$)$. Equation 4.17 ) gives the closest values. Then, by comparing the improved ISPT expression to the original SPT equation, taking into account the fact that the term $\ln \mathcal{G}(\sigma)$ related to connectivity is of the same order of magnitude in the both cases, we deduce that the main correction incorporated in equation (4.16), with respect to equation (4.12), is made to the reference term of dissociated hard spheres.

\section{Alternative relations}

The results represented in the previous table suggest the possibility of developing new expressions, allowing us to describe first dumbbells made of tangent spheres, and then those with overlap. So far, relationships between conformal and TPT theories have been presented. On the other hand, the contact pair distribution function is known to be better described by the expression deduced from the SPT than by that obtained with the Ornstein Zernike equation and the PY approximation. Then, it was first looked for possible alternative relationships between the expression of the pressure deduced from the WOZ integral equations and the expression of the contact pair distribution function deduced from the SPT. 
Table 1. Comparison between various expressions of $\ln \mathcal{G}(\sigma)$, given by equations 4.13 , , 4.17) and 4.19), with $\ln g_{12}^{\mathrm{SPT}}\left(\sigma_{12}\right)$ deduced from 4.14 , as a function of the volume fraction $\zeta_{3}$, when $\sigma_{1}=\sigma_{2}$.

\begin{tabular}{|c|c|c|c|c|}
\hline$\zeta_{3}$ & $\ln \mathcal{G}_{\text {SPT }}(\sigma)$ & $\ln \mathcal{G}_{\text {ISPT }}(\sigma)$ & $\ln g_{12}^{\text {SPT }}(\sigma)$ & $\ln \mathcal{G}_{\text {SFP }}(\sigma)$ \\
\hline \hline 0.05 & 0.1292 & 0.1292 & 0.1292 & 0.1302 \\
\hline 0.1 & 0.2674 & 0.2675 & 0.2674 & 0.2720 \\
\hline 0.15 & 0.4155 & 0.4160 & 0.4157 & 0.4272 \\
\hline 0.2 & 0.5747 & 0.5758 & 0.5751 & 0.5981 \\
\hline 0.25 & 0.7460 & 0.7485 & 0.7472 & 0.7877 \\
\hline 0.3 & 0.9307 & 0.9356 & 0.9336 & 0.9995 \\
\hline 0.35 & 1.1297 & 1.1391 & 1.1364 & 1.2385 \\
\hline 0.4 & 1.3442 & 1.3608 & 1.3581 & 1.5108 \\
\hline 0.45 & 1.5741 & 1.6027 & 1.6019 & 1.8251 \\
\hline 0.5 & 1.8181 & 1.8664 & 1.8718 & 2.1931 \\
\hline
\end{tabular}

\subsection{Equations deduced from integral equations}

In this framework, it was observed the equality of equation (4.8) of the SFP theory with expression (2.6b) obtained independently with the WOZ integral equations. Then, it seems also interesting to find possible relations between the equations of the TPT and those deduced from integral equations. The getting of the expression 2.6b) from the equation (2.6a), has been reconsidered, in order to obtain an alternative expression, by assuming that the contact pair distribution function is given by the SPT equation. Previously, from equation (2.6a) the relation 2.5 defining the pair concentration $\rho_{\mathrm{D}}$ was used to arrive at equation 2.6b. The equality between $g_{12}^{00}\left(\sigma_{12}\right)$ and $g_{12}^{\mathrm{PY}}\left(\sigma_{12}\right)$ was also used. Now, it is assumed that $g_{12}^{00}\left(\sigma_{12}\right)$ is equal to $g_{12}^{\mathrm{SPT}}\left(\sigma_{12}\right)$ in the equation 2.5 defining $\rho_{\mathrm{D}}$ the concentration of pairs:

$$
\rho_{\mathrm{D}}=\rho_{1}^{0} \rho_{2}^{0} K^{0} g_{12}^{\mathrm{SPT}}\left(\sigma_{12}\right) \text {. }
$$

This definition is an improvement because the expression of the pair distribution function at the contact given by SPT is known to be better than that given by the PY approximation. On the other hand, the equality between $g_{12}^{00}\left(\sigma_{12}\right)$ and $g_{12}^{\mathrm{PY}}\left(\sigma_{12}\right)$ is kept in equation 2.6a), because $g_{12}^{00}\left(\sigma_{12}\right)$ is effectively found to be given by the right-hand part of the equation 2.7 in the framework of the WOZ equations. The use of the new definition of the concentration of pair $\rho_{\mathrm{D}}$, then leads, starting from equation (2.6a), by expressing $\rho_{1}^{0} \rho_{2}^{0} K^{0}$ as a function of $\rho_{\mathrm{D}}$, to the alternative expression

$$
\beta P=\beta P_{\mathrm{PY}}^{c}-\rho_{\mathrm{D}} \frac{\left[g_{12}^{\mathrm{PY}}\left(\sigma_{12}\right)\right]^{2}}{g_{12}^{\mathrm{SPT}}\left(\sigma_{12}\right)} .
$$

Otherwise, inspired by equation (7.19) seen in [4], the following relation between $\left[g_{12}^{\mathrm{PY}}\left(\sigma_{12}\right)\right]^{2}, g_{12}^{\mathrm{SPT}}\left(\sigma_{12}\right)$ and its derivatives was found

$$
g_{12}^{\mathrm{SPT}}\left(\sigma_{12}\right)+\sum_{k} \rho_{k} \frac{\partial g_{12}^{\mathrm{SPT}}\left(\sigma_{12}\right)}{\partial \rho_{k}}=\left[g_{12}^{\mathrm{PY}}\left(\sigma_{12}\right)\right]^{2} .
$$

Therefore, by multiplying by $\rho_{1}^{0} \rho_{2}^{0} K^{0}$ on both sides, the following equality is obtained

$$
\rho_{\mathrm{D}} \frac{\left[g_{12}^{\mathrm{PY}}\left(\sigma_{12}\right)\right]^{2}}{g_{12}^{\mathrm{SPT}}\left(\sigma_{12}\right)}=\rho_{\mathrm{D}}\left[1+\sum_{k} \rho_{k} \frac{\partial \ln \left[g_{12}^{\mathrm{SPT}}\left(\sigma_{12}\right)\right]}{\partial \rho_{k}}\right],
$$


with $\rho_{\mathrm{D}}$ given by $(5.1)$. The left part of the previous equation appears in equation $(5.2)$ and, in view of equations (2.12), the right part is the relation used in the TPT to calculate the connectivity part of the pressure when $g^{\text {ref }}\left(\sigma_{12}\right)$, the reference pair distribution function, is given by $g_{12}^{\mathrm{SPT}}\left(\sigma_{12}\right)$. Therefore, the relation (5.2), deduced from the WOZ integral equations, can be obtained independently within TPT. Since the SPT expression of the contact pair distribution function is better than that given by the PY approximation, the equation (5.2) is probably an improvement over (2.6b). However, the expression of the reference pair distribution at contact with $g_{12}^{\mathrm{SPT}}\left(\sigma_{12}\right)$ is known to be slightly poorer than that given by the corresponding BM equation. Accordingly, the equation (5.2) is probably somewhat less efficient than the corresponding TPT equation obtained using the BM approximation.

\subsection{Equations deduced from conformal theories}

It has been seen in table 1 , that, when $l=\sigma_{12}$, the connectivity term given in the expression of the improved free energy ISPT is numerically close to the one found for the initial free energy SPT. The improvement present in the ISPT equations is provided by the first term describing the dissociated hard spheres. The effect of connectivity is taken into account using a simpler equation in the initial SPT theory.

In view of these observations, an alternative expression, providing a correction to the SPT expression of the free energy (4.12), when $l=\sigma_{12}$, was deduced. A modification can be made to the SPT expression by only changing the term of dissociated hard spheres. Then, when $l=\sigma_{12}$, a new equation for the free energy can be obtained:

$$
\beta \frac{A_{\mathrm{New}}\left(l=\sigma_{12}\right)}{V}=\beta \frac{A_{\mathrm{BM}}}{V}-\rho_{\mathrm{D}} \ln \mathcal{G}_{\mathrm{SPT}}\left(\sigma_{12}\right) .
$$

Compared with equation (4.12), the first term $A_{\mathrm{PY}}^{c}$ is replaced by a more accurate term $A_{\mathrm{BM}}$, and the second term remains unchanged because it leads to numerical values close to that given by the corresponding term of the equation 4.16 . The corresponding expression of the pressure is obtained by differentiation of the free energy:

$$
\beta P_{\mathrm{New}}=\beta P_{\mathrm{BM}}-\rho_{\mathrm{D}}\left[g_{12}^{\mathrm{PY}}\left(\sigma_{12}\right)-\frac{3 \zeta_{2}^{2}}{\left(1-\zeta_{3}\right)^{3}} \frac{\sigma_{1}^{2} \sigma_{2}^{2}}{\left(\sigma_{1}+\sigma_{2}\right)^{2}}\right] \quad \text { for: } \quad l=\sigma_{12} .
$$

This relation allows us to correctly describe the thermodynamics of dumbbells made of tangent spheres. As such, it is of little use insofar as many expressions are already available. Nevertheless, from this equation a more general relation can be established to describe dimers with overlapping spheres. Indeed, equations (5.5) and (5.6) are linear combinations of various relations previously introduced

$$
\begin{aligned}
& \beta A_{\mathrm{New}}=\beta A_{\mathrm{ISFP}}+\beta\left[A_{\mathrm{SPT}}-A_{\mathrm{SFP}}\right], \\
& \beta P_{\mathrm{New}}=\beta P_{\mathrm{ISFP}}+\beta\left[P_{\mathrm{SPT}}-P_{\mathrm{SFP}}\right] .
\end{aligned}
$$

These new relationships add corrective terms to the previous ISFP equations. Moreover, these latter expressions can also be used for dimers with overlapping of the spheres by using the more general equations introduced previously to describe them. Therefore, using equations (3.4), 3.1) and (3.3), the equation (5.7b) has been evaluated for a dumbbell fluid where the distance $l$ between sphere is less than or equal to 1 . The term in square brackets, $\left[P_{\mathrm{SPT}}-P_{\mathrm{SPP}}\right]$ in the previous equation, can be simplified, giving

$$
\beta P_{\mathrm{New}}=\beta P_{\mathrm{ISFP}}+\frac{s^{2}}{3(1-v)^{3}}\left(q-\frac{s}{4 \pi}\right),
$$

where the difference $q-s /(4 \pi)$, starting from equations 4.2 ) and 4.3 , can be evaluated according to

$$
\left(q-\frac{s}{4 \pi}\right)=\frac{\rho_{\mathrm{D}}}{16}\left[l-\frac{\left(\sigma_{1}-\sigma_{2}\right)^{2}}{4 l}\right]^{2} .
$$

The results of the calculations performed with equation (5.8) are presented in figure 2, for homonuclear dimers, for various separations of the constituents. A comparison is also given with the results deduced 


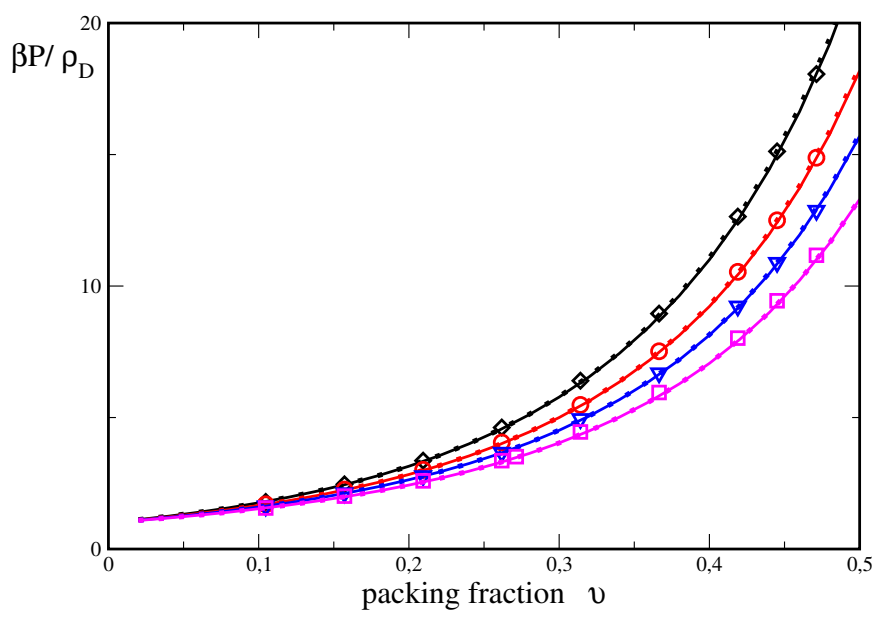

Figure 2. (Colour online) Equation of state for overlapping hard sphere dumbbells. From top to bottom, lines represent theoretical results for different overlap distances: $\cdots$ New equation $(5.8),-$ ISPT equation (3.2). Symbols are the simulation results [53]. Diamonds and black lines: $l=1$; Circles and red lines: $l=0.8$; Triangles and blue lines: $l=0.6$, Squares and pink lines: $l=0.2$.

from the ISPT equation (3.2). It is observed that the new theoretical expression describes well the values obtained by simulation. It provides values numerically very close to those given by the ISPT equation. The new equation can be seen also as an improvement of the improved SFP equation (3.4). The corrective term for $P_{\mathrm{ISFP}}$, presented in equation (5.8), seems to correspond numerically to the contribution required to recover the results obtained with the expression of $P_{\text {ISPT }}$.

\section{Conclusion}

In the previous sections, it has been shown how, from the conformal theories applied to dumbbells made of tangent hard spheres, the pressure and the free energy can be split into a term of repulsion of hard spheres and a term of connectivity between these spheres. This separation is similar to that encountered in the expression of pressure in the TPT theory. The analysis presented in this work highlights the relative influence of these two contributions in the different expressions presented in various conformal theories studied. It has been found that for each class of conformal theory the reference term is, for the original version that given by the PY theory, and for the improved version that given by the BMCSL expression. Then, when the scaled particle and the scaled field theory are compared, it was noted that the terms of reference are the same, both for the original approach and for the improved expressions. On the other hand, for each of the two classes of conformal theory considered, the term of connectivity changes little when one goes from the original expression to the improved one. In addition, the term of connectivity is different between the scaled field and the scaled particle theory. Connections and similarities have also been seen between the expressions describing these two contributions and those found with the TPT for dimers made of tangent spheres. Similarities were also found with some relationships given in the context of integral equations. This work allows one to explain differences found in the description of thermodynamic properties deduced from simulations.

Furthermore, this analysis has allowed one to provide alternative expressions of the connectivity terms, in particular for dimers with overlap. It could be extended to fluids containing chains of a greater number of constituents. It could also be useful to better represent subunits belonging to polymers according to the group contribution formalism [56]. On the other hand, the analysis of thermodynamic quantities such as free energy and pressure could be extended to the activity coefficients of various species present in the 
fluids of dimers [57]. Then, the study of the chemical equilibrium between the hard spheres forming the overlapping dimers could be reviewed. Thus, this will allow one to describe the background correlation in continuation of previous studies on this subject [58-60].

\section{References}

1. Helfand E., Reiss H., Frisch H. L., Lebowitz J. L., J. Chem. Phys., 1960, 33, 1379-1385, doi 10.1063/1.1731417

2. Boublík T., J. Chem. Phys., 1975, 63, 4084, doi 10.1063/1.431882

3. Barboy B., Gelbart W. M., J. Chem. Phys., 1979, 71, 3053-3062, doi $10.1063 / 1.438711$

4. Rosenfeld Y., J. Chem. Phys., 1988, 89, 4272-4287, doi 10.1063/1.454810.

5. Carnahan N. F., Müller E. A., Pikunic J., Phys. Chem. Chem. Phys., 1999, 1, 4259-4266, doi:10.1039/A903338F

6. Oversteegen S. M., Roth R., J. Chem. Phys., 2005, 122, 214502, doi:10.1063/1.1908765

7. Rosenfeld Y., Phys. Rev. Lett., 1989, 63, 980-983, doi:10.1103/PhysRevLett.63.980

8. Wertheim M. S., J. Stat. Phys., 1984, 35, 19-34, doi:10.1007/BF01017362

9. Wertheim M. S., J. Stat. Phys., 1984, 35, 35-47, doi:10.1007/BF01017363

10. Chapman W. G., Gubbins K. E., Jackson G., Radosz M., Fluid Phase Equilib., 1989, 52, 31-38, doi $10.1016 / 0378-3812(89) 80308-5$

11. Barker J. A., Henderson D., J. Chem. Phys., 1967, 47, 2856-2861, doi $10.1063 / 1.1712308$

12. Barker J. A., Henderson D., J. Chem. Phys., 1967, 47, 4714-4720, doi $10.1063 / 1.1701689$.

13. Weeks J. D., Chandler D., Andersen H. C., J. Chem. Phys., 1971, 54, 5237-5247, doi:10.1063/1.1674820

14. Andersen H. C., Weeks J. D., Chandler D., Phys. Rev. A, 1971, 4, 1597-1606, doi 10.1103/PhysRevA.4.1597.

15. Ornstein L. S., Zernike F., Koninklijke Nederlandsche Akademie van Wetenschappen Proceedings, 1914, 17, 793.

16. Percus J. K., Yevick G. J., Phys. Rev., 1958, 110, 1-13, doi: $10.1103 /$ PhysRev.110.1

17. Wertheim M. S., Phys. Rev. Lett., 1963, 10, 321-322, doi $10.1103 /$ PhysRevLett.10.321.

18. Thiele E., J. Chem. Phys., 1963, 39, 474-478, doi $10.1063 / 1.1734272$

19. Lebowitz J. L., Phys. Rev., 1964, 133, A895-A899, doi:10.1103/PhysRev.133.A895

20. Hansen J.-P., McDonald I. R., Theory of Simple Liquids, Academic Press, Amsterdam, 2006.

21. Lebowitz J. L., Rowlinson J. S., J. Chem. Phys., 1964, 41, 133-138, doi:10.1063/1.1725611

22. Baxter R. J., J. Chem. Phys., 1970, 52, 4559-4562, doi 10.1063/1.1673684.

23. Salacuse J. J., Stell G., J. Chem. Phys., 1982, 77, 3714-3725, doi:10.1063/1.444274

24. Carnahan N. F., Starling K. E., J. Chem. Phys., 1969, 51, 635-636, doi:10.1063/1.1672048

25. Boublík T., J. Chem. Phys., 1970, 53, 471-472, doi $10.1063 / 1.1673824$

26. Mansoori G. A., Carnahan N. F., Starling K. E., Leland T. W., J. Chem. Phys., 1971, 54, 1523-1525, doi $10.1063 / 1.1675048$

27. Chandler D., Silbey R., Ladanyi B. M., Mol. Phys., 1982, 46, 1335-1345, doi $10.1080 / 00268978200101971$

28. Rossky P. J., Chiles R. A., Mol. Phys., 1984, 51, 661-674, doi:10.1080/00268978400100451.

29. Wertheim M. S., J. Stat. Phys., 1986, 42, 459-476, doi:10.1007/BF01127721

30. Wertheim M. S., J. Stat. Phys., 1986, 42, 477-492, doi:10.1007/BF01127722

31. Wertheim M. S., J. Chem. Phys., 1986, 85, 2929-2936, doi:10.1063/1.451002

32. Wertheim M. S., J. Chem. Phys., 1987, 87, 7323-7331, doi $10.1063 / 1.453326$

33. Kalyuzhnyi Yu. V., Stell G., Mol. Phys., 1993, 78, 1247-1258, doi:10.1080/00268979300100821

34. Yakub E. S., Mol. Phys., 1992, 77, 845-855, doi 10.1080/00268979200102821

35. Kalyuzhnyi Yu. V., Protsykevitch I. A., Holovko M. F., Chem. Phys. Lett., 1993, 215, 1-4, doi $10.1016 / 0009-2614(93) 89253-E$

36. Kalyuzhnyi Yu. V., Stell G., Llano-Restrepo M. L., Chapman W. G., Holovko M. F., J. Chem. Phys., 1994, 101, 7939-7952, doi $10.1063 / 1.468221$

37. Chiew Y. C., Mol. Phys., 1990, 70, 129-143, doi $10.1080 / 00268979000100891$

38. Kalyuzhnyi Yu. V., Cummings P. T., J. Chem. Phys., 1999, 105, 2011-2019, doi: 10.1063/1.472069.

39. Stell G., Lin C.-T., Kalyuzhnyi Yu. V., J. Chem. Phys., 1999, 110, 5444-5457, doi:10.1063/1.478440

40. Stell G., Lin C.-T., Kalyuzhnyi Yu. V., J. Chem. Phys., 1999, 110, 5458-5468, doi:10.1063/1.478441

41. Jackson G., Chapman W. G., Gubbins K. E., Mol. Phys., 1988, 65, 1-31, doi $10.1080 / 00268978800100821$

42. Chapman W. G., Jackson G., Gubbins K. E., Mol. Phys., 1988, 65, 1057-1079, doi $10.1080 / 00268978800101601$.

43. Archer A. L., Jackson G., Mol. Phys., 1991, 73, 881-896, doi:10.1080/00268979100101631

44. Boublík T., Mol. Phys., 1989, 68, 191-198, doi 10.1080/00268978900102051

45. Walsh J. M., Gubbins K. E., J. Phys. Chem., 1990, 94, 5115-5120, doi $10.1021 /$ j100375a065 
46. Boublík T.,Vega C., Diaz-Peña M., J. Chem. Phys., 1990, 93, 730-736, doi: $10.1063 / 1.459523$

47. Amos M. D., Jackson G., J. Chem. Phys., 1992, 96, 4604-4618, doi 10.1063/1.462796

48. Lebowitz J. L., Helfand E., Praestgaard E., J. Chem. Phys., 1965, 43, 774-778, doi:10.1063/1.1696842

49. Gibbons R. M., Mol. Phys., 1969, 17, 81-86, doi:10.1080/00268976900100811

50. Gibbons R. M., Mol. Phys., 1970, 18, 809-816, doi:10.1080/00268977000100871

51. Boublík T., Mol. Phys., 1974, 27, 1415-1427, doi:10.1080/00268977400101191

52. Boublík T., Nezbeda I., Chem. Phys. Lett., 1977, 46, 315-316, doi 10.1016/0009-2614(77)85269-X

53. Tildesley D. J., Streett W. B., Mol. Phys., 1980, 41, 85-94, doi 10.1080/00268978000102591

54. Olaussen K., Stell G., J. Stat. Phys., 1991, 62, 221-237, doi:10.1007/BF01020867

55. Fisher M. E., Zuckerman D. M., J. Chem. Phys., 1998, 109, 7961-7981, doi:10.1063/1.477444

56. Lymperiadis A., Adjiman C. S., Jackson G., Galindo A., Fluid Phase Equilib., 2008, 274, 85-104, doi $10.1016 / j$.fluid.2008.08.005.

57. Labík S., Jirácek V., Malijevský A., Smith W. R., Chem. Phys. Lett. 1995, 247, 227-231, doi $10.1016 / 0009-2614(95) 01214-0$

58. Boublík T., Mol. Phys., 1986, 59, 775-793, doi 10.1080/00268978600102391

59. Labík S., Smith W. R., J. Chem. Phys., 1988, 88, 1223-1227, doi:10.1063/1.454242

60. Boublík T., Mol. Phys., 2006, 104, 3425-3433, doi:10.1080/00268970601014831

\section{Взаємозв'язок між термодинамічними збуреннями та теоріями масштабованої частинки для конденсованих рідких димерів}

\section{О. Бернар}

Лабораторія PHENIX, CNRS, Університет Сорбонни (Кампус П'єра та Марії Кюрі), 4 Плас Жюссіє, F-75005

Париж, Франція

У роботі зроблено огляд різних підходів для побудови теорії масштабованої частинки з метою опису плинів гантелеподібних частинок, утворених з твердих сфер, що перекриваються або торкаються одна одної. Надано інше представлення відомим з літератури виразам у математичній формі, подібній до тієї, яка відома з термодинамічної теорії збурень, отриманої Вертгаймом для ланцюжків і узагальненої у статистичній теорії асоціативних рідин. Аналогії та відмінності між цими двома теоретичними підходами дозволили запропонувати альтернативні вирази для опису плинів гантелеподібних частинок зі сферами, що перекриваються.

Ключові слова: плини гантелеподібних частинок з твердими серцевинами, теорія масштабованоі частинки, термодинамічна теорія збурень 


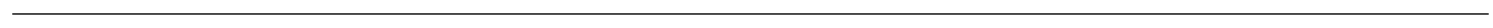

